\title{
Biogenesis of a Solid Condensate: Amyloid bodies
}

\author{
Miling Wang ${ }^{1,2}$ and Stephen Lee $e^{1,2,3,4^{*}}$ \\ 1 Department of Biochemistry and Molecular Biology, Miller School of Medicine, University of Miami, \\ Miami, FL 33136, USA \\ 2 Sylvester Comprehensive Cancer Center, Miller School of Medicine, University of Miami, Miami, FL 33136, \\ USA \\ 3 Department of Urology, Miller School of Medicine, University of Miami, FL 33136, USA \\ * Correspondence: stephenlee@med.miami.edu
}

\begin{abstract}
This year marks the 20th anniversary of the discovery that proteins with various cellular functions can be temporarily immobilized in the nucleolus, a process known as nucleolar sequestration. This review reflects on the progress made to understand the physiological roles of nucleolar sequestration and the mechanisms involved in protein immobilization. We discuss how nucleolar sequestration consists of a highly choreographed amyloidogenic liquid-to-solid phase transition that converts the nucleolus into Amyloid bodies (A-bodies). The study of solid condensates A-bodies will offer unique perspectives on cellular assembly of membrane-less compartments and provide alternative insights on pathological amyloidogenesis involved in neurological disorders.
\end{abstract}

Keywords: Nucleolar sequestration; nucleolus; physiological amyloids; ribosomal intergenic spacer; rIGSRNA; liquid-liquid phase separation; liquid-to-solid phase transition; complex coacervation; biomolecular condensates; beta-amyloid; MDM2; VHL; Cdc14; low complexity RNA; acidosis; heat shock

\section{Nucleolar sequestration: Visitors to the nucleolus}

The role of the nucleolus as the site of ribosome biosynthesis was established by the mid-1960's [1-3]. Nucleoli are built around tandem repeats of ribosomal DNA (rDNA) in nucleolar organizing regions (NORs) and structurally dependent on active transcription of rDNA [4,5]. The traditional role of the nucleolus as a hub of rRNA synthesis and ribosome assembly has been the subject of many excellent literature surveys [6-8]. This review focuses on a lesser known phenomenon originally coined "nucleolar sequestration", which describes the nucleolus' ability to sequester and release regulatory proteins in response to cellular cues [9-11]. 2019 will mark 20 years since nucleolar sequestration was first hypothesized by Bachant and Elledge [12] following the discoveries that cellcycle regulator Cdc14 phosphatase and E3 ubiquitin ligase MDM2 could be temporarily localized in nucleoli to affect cell-cycle progression [13-17]. In S. cerevisiae, Cdc14 is sequestered in the nucleolus by Cfi1/Net1 until the onset of anaphase, when it is liberated to promote mitosis $[15,16]$. In mammalian cells, nucleolar sequestration of MDM2 prevents it from binding and exporting p53 into the cytoplasm for degradation $[13,14,17]$. This stabilizes p53 in the nucleus, where it acts as a transcription factor that promotes cell-cycle arrest or apoptosis. Another notable example of a mammalian protein is tumor suppressor VHL (von Hippel-Lindau protein), which is sequestered in the nucleolus in response to hypoxia-induced acidosis [18]. E3 ubiquitin ligase VHL degrades transcription factor HIF (hypoxia-inducible factor) under normal oxygen conditions. Nucleolar sequestration of VHL in hypoxic low $\mathrm{pH}$ settings enables HIF to evade degradation and activate its target genes involved in oxygen homeostasis. Cdc14, MDM2 and VHL represent three of the many examples reported over the last two decades. Table 1 summarizes the reports of nucleolar sequestration that have been observed for many other proteins under various cellular conditions, 
including development [19-21], apoptosis [22,23], DNA damage [17,24,25], extracellular acidosis [18,26-28], heat shock [26-30], UV and ionizing radiation [31,32], alcohol [33], viral infection [34], transcriptional arrest [35], inhibition of rRNA synthesis [36] and proteasomal inhibition [29,37-39].

\section{Nucleolar sequestration: A case of protein immobilization}

Table 1. List of the proteins whose activities have been reported to be regulated by nucleolar sequestration.

\begin{tabular}{|c|c|c|c|c|}
\hline $\begin{array}{l}\text { Protein } \\
\text { symbol }\end{array}$ & Full name & Stimulus & $\begin{array}{l}\text { Nucleolar } \\
\text { response }\end{array}$ & Reference \\
\hline Cdc14 & $\begin{array}{l}\text { Cell division cycle } 14 \\
\text { (S. cerevisiae) }\end{array}$ & Anaphase & Release & $\begin{array}{c}\text { Shou et al., } \\
1999 \\
\text { Visintin et al., } \\
1999\end{array}$ \\
\hline Pch2 & $\begin{array}{c}\text { Pachytene checkpoint } 2 \\
\text { (S. cerevisiae) }\end{array}$ & $\begin{array}{c}\text { Meiotic prophase } \\
\text { arrest }\end{array}$ & Release & $\begin{array}{c}\text { San-Segundo } \\
\text { and Roeder, } \\
1999 \\
\end{array}$ \\
\hline MDM2 & $\begin{array}{l}\text { Murine double minute } \\
22 \text { homolog }\end{array}$ & $\begin{array}{l}\text { Ribosomal stress } \\
\text { DNA damage }\end{array}$ & Capture & $\begin{array}{c}\text { Weber et al., } \\
1999 \\
\text { Lohrum et al., } \\
2000 \\
\text { Bernardi et al., } \\
2004 \\
\end{array}$ \\
\hline \multirow[t]{2}{*}{ hTERT } & \multirow[t]{2}{*}{$\begin{array}{l}\text { Human telomerase } \\
\text { reverse transcriptase }\end{array}$} & $\begin{array}{l}\text { Transformation, DNA } \\
\text { damage }\end{array}$ & Release & \multirow[t]{2}{*}{$\begin{array}{l}\text { Wong et al., } \\
2002\end{array}$} \\
\hline & & Ionizing radiation & Capture & \\
\hline c-Myc & Proto-oncogene c-Myc & Proteasomal stress & Capture & Arabi, 2003 \\
\hline ADAR2 & $\begin{array}{c}\text { Adenosine deaminase } \\
\text { that acts on RNA } 2\end{array}$ & $\begin{array}{c}\text { Inhibition of rRNA } \\
\text { synthesis }\end{array}$ & Release & $\begin{array}{c}\text { Sansam et al., } \\
2003 \\
\end{array}$ \\
\hline VHL & $\begin{array}{l}\text { von Hippel Lindau } \\
\text { tumor suppressor }\end{array}$ & Extracellular acidosis & Capture & $\begin{array}{l}\text { Mekhail et al., } \\
2004\end{array}$ \\
\hline RelA & $\begin{array}{l}\text { p65 subunit of } \\
\text { transcription factor } \\
\text { NF-кB }\end{array}$ & $\begin{array}{l}\text { Aspirin, serum } \\
\text { withdrawal, UV-C } \\
\text { radiation }\end{array}$ & Capture & $\begin{array}{c}\text { Stark and } \\
\text { Dunlop, } 2005 \\
\text { Chen and } \\
\text { Stark, } 2017\end{array}$ \\
\hline Polycomb & $\begin{array}{c}\text { Polycomb } \\
\text { (D. melanogaster) }\end{array}$ & Cell differentiation & Capture & $\begin{array}{l}\text { Chen et al., } \\
2005 \\
\end{array}$ \\
\hline Hand1 & $\begin{array}{l}\text { Heart and neural crest } \\
\text { derivatives expressed } 1\end{array}$ & Cell differentiation & $\begin{array}{l}\text { Capture/ } \\
\text { Release }\end{array}$ & $\begin{array}{c}\text { Martindill et } \\
\text { al., } 2007\end{array}$ \\
\hline Hsc70 & $\begin{array}{c}\text { Heat shock chaperone } \\
70 \\
\end{array}$ & $\begin{array}{c}\text { Recovery from heat } \\
\text { shock }\end{array}$ & Capture & $\begin{array}{c}\text { Banski et al., } \\
2010 \\
\end{array}$ \\
\hline Ulp1 & $\begin{array}{c}\text { Small ubiquitin-related } \\
\text { modifier (SUMO) } \\
\text { protease (S. cerevisiae) }\end{array}$ & Alcohol & Capture & $\begin{array}{c}\text { Sydorskyy et } \\
\text { al., } 2010\end{array}$ \\
\hline p53 & $\begin{array}{l}\text { Cell-cycle regulator; } \\
\text { tumor suppressor }\end{array}$ & $\begin{array}{c}\text { Proteasomal } \\
\text { inhibition (MG132) }\end{array}$ & Capture & $\begin{array}{c}\text { Krüger and } \\
\text { Scheer, } 2010 \\
\text { Latonen et al., } \\
2011\end{array}$ \\
\hline Piwi & $\begin{array}{c}\text { piRNA binding protein } \\
(\text { Drosophila })\end{array}$ & Heat shock & Capture & $\begin{array}{c}\text { Mikhaleva et } \\
\text { al., } 2018 \\
\end{array}$ \\
\hline
\end{tabular}


Much work has been devoted to study the high mobility of proteins in the cell, particularly within the nucleus [40,41]. Typically, proteins maintain their dynamic profiles as they rapidly exchange between affinity interactions and the cellular milieu, turning over within seconds to minutes [41-43]. The nucleolus is considered a dynamic droplet, assembled by liquid-liquid phase separation [44,45], whose proteins are highly mobile [35]. It is remarkable, then, that proteins sequestered in the nucleolus are immobile, as assessed by photobleaching analyses and heterokaryon fusion studies [18,27,28,46]. This has been demonstrated for Cdc14 [47], MDM2 [27], VHL [46], RNF8 [48], DNMT1 [27], and a recently reported visitor, piRNA binding protein Piwi in Drosophila under heat shock [49]. Even resident nucleolar proteins such as RNA polymerase I subunit RPA16, Pescadillo and SENP3 can undergo cycles of mobility/immobility [28]. Here, VHL is used to elaborate the dynamics involved: VHL switches from a highly dynamic, uniform distribution under standard growth conditions $\left(21 \% \mathrm{O}_{2}, \mathrm{pH} 7.4\right)$ to an immobilized state in the nucleolus rapidly on exposure to extracellular acidosis $\left(1 \% \mathrm{O}_{2}, \mathrm{pH}\right.$ 6.0) [46]. Only upon neutralization of extracellular $\mathrm{pH}$ is VHL released from the nucleolus to return to its original distribution and mobility [46]. These immobile nuclear foci were originally called "nucleolar detention centers", as targets are both localized and detained within the nucleolus, unable to freely diffuse elsewhere $[28,46,50]$. From this perspective, the function of nucleolar detention or immobilization is to temporarily inactivate relevant proteins, as they would be kept away from their normal targets. Just as possible, though, is that the clustering of detained proteins may render an enzymatic reaction more efficient. Reports suggest that immobilized nucleolar Cdc14 maintains Spo12 dephosphorylation [47] and Piwi switches from its canonical role in non-nucleolar transposable element (TE) repression to rDNA-specific TE repression when it is immobilized in the nucleolus [49]. From these studies, it is clear that cells have evolved a strategy to regulate molecular networks by reversibly switching proteins between a mobile and an immobile state. Whether nucleolar sequestration represents a loss- or gain-of-function might depend on which proteins undergo immobilization.

\section{Mechanisms of nucleolar sequestration}

MDM2, Cdc14 and VHL are examples where further work has yielded mechanistic insights as to how proteins are sequestered in the nucleolus. MDM2 exhibits flexibility in how it becomes localized in the nucleolus. Under conditions where tumor suppressor $\mathrm{p}^{14 / 19} \mathrm{ARF}$ is induced, ARF binding MDM2 unmasks a cryptic nucleolar localization signal (NoLS) within its C-terminal RING domain, which is essential for MDM2 nucleolar sequestration [14,17,51,52]. Nucleotide binding, specifically ATP, to MDM2 behaves similarly, independent of ARF [53]. Alternatively, during DNA damage, e.g. doxorubicin, and acidosis, MDM2 is shuttled into the nucleolus by direct binding to PML (promyelocytic leukemia protein) [13], itself a target of nucleolar sequestration [54]. Cdc14 is anchored in the nucleolus for most of the cell cycle through its association with Cfi1/Net1 [15,16]. The timing of its release during cell division is regulated by a signaling cascade of phosphorylation events that directly weaken the interaction of Cdc14 with Net1, releasing Cdc14 to act as a mitotic exit activator [55]. Whether these targeting signals also promote protein immobilization is unclear. Mapping analysis of VHL identified an approximately 30 amino acid fragment referred to as a nucleolar detention signal (NoDS) that is necessary and sufficient to immobilize proteins in nucleoli [48]. The NoDS is composed of an arginine/histidine-rich sequence followed by two or more hydrophobic LXV motifs where X can be any hydrophobic residue e.g. LWL, LLV, LFV, LQV [48]. The NoDS is highly predictive and was identified in many other candidates including DNA methyltransferase I DNMT1, promyelocytic leukemia protein PML and DNA polymerase delta catalytic subunit POLD1, all shown to be immobilized in nucleoli under extracellular acidosis [27,48]. The identification of the NoDS in many proteins provided evidence that nucleolar sequestration is a common cellular strategy to regulate protein function. 


\section{Transformation of nucleoli into Amyloid bodies (A-bodies)}

Proteins with limited mobility have been observed to display amyloid-like properties in physiological settings [56,57]. Amyloidogenesis is the process whereby soluble proteins assemble into aggregates known as amyloid fibrils [58]. Nucleolar detention centers composed of proteins such as VHL, MDM2, POLD1, etc share many properties associated with the amyloid state beyond immobility. First, these nuclear foci stained positive with amyloidophilic dyes such as Congo red, Thioflavin S/T, Amylo-Glo, and ANS [26]. Second, the fibrillar organization of these nuclear foci observable by electron microscopy (EM) are as predicted for the amyloid-fold [26]. Third, proteins found in these nuclear foci displayed biochemical properties associated with amyloids, including resistance to Proteinase $\mathrm{K}$ and insolubility in common detergents [26]. Consequently, the term "nucleolar detention centers" was replaced with "Amyloid bodies" (A-bodies) to reference the transformation of the nucleolus into an A-body, a molecular prison of proteins in their amyloid-like state [26].

\section{Amyloid bodies are distinct from liquid-like membrane-less compartments}

Amyloid-like biophysical characteristics make A-bodies distinct from other membrane-less compartments that display liquid-like properties [44,45,59-64], such as stress granules, nucleoli and germ granules, and from other structures that originate from the nucleolus (e.g. nucleolar caps and nucleolar aggresomes). Liquid-like membrane-less compartments are dynamic, their constituents are mobile and they do not typically stain with amyloidophilic dyes [41,59]. Thus, A-bodies are better categorized as solid-like structures as they are static and harbor immobile proteins that form strong intermolecular bonds associated with the amyloid-fold. Several authors have suggested that the formation of A-bodies represents a physiological liquid-to-solid phase transition rather than a liquidliquid phase separation implicated in the formation of liquid condensates $[65,66]$. The term liquid-tosolid phase transition had, thus far, been reserved to describe the formation of pathological aggregates [67-72]. Other physiological examples of solid-like structures include Balbiani bodies observed in Xenopus [73] and pH-regulated fluid-to-solid transition of the cytoplasm in yeast [74]. Table 2 summarizes the differences between liquid-like and solid-like condensates in the cell. By similar criteria, A-bodies may be contrasted from other stress-induced nucleolar structures, namely nucleolar caps [75] and nucleolar aggresomes [37,38]. By EM, A-bodies have a unique fibrillar organization characteristic of amyloids that has yet to be observed in the cell [26] (Fig. 1). Nucleolar caps appear as electron-dense amorphous structures and nucleolar aggresomes are cavernous, occupying a large electron-light central space of the nucleolus [76] (Fig. 1). Each structure exhibits significant remodeling of the nucleolus $[28,75,76]$. Interestingly, while the formation of nucleolar caps and A-bodies is accompanied by a redistribution of nucleolar components and subsequent arrest of ribosomal biogenesis [28,75,77], nucleolar aggresomes require transcriptionally active nucleoli to form $[37,38]$ with all nucleolar compartments intact and visible by EM. Additionally, proteins are dynamically sorted into nucleolar caps and remain mobile [75] while proteins in A-bodies are immobile [26-28,46]. Photobleaching experiments have not been reported for proteins of nucleolar aggresomes. Therefore, A-bodies are unique structures with amyloidogenic properties distinct from other cellular bodies. 
Table 2. Contrast between liquid-like and solid-like condensates in the cell.

\begin{tabular}{|c|c|c|}
\hline & Liquid-like condensates & Solid-like condensates \\
\hline Examples & $\begin{array}{l}\text { Cytoplasm: } \\
\text { - Stress granules } \\
\text { - P-bodies } \\
\text { Nuclear: } \\
\text { - Stress granules } \\
\text { - } \quad \text { Cajal bodies } \\
\text { - Speckles } \\
\text { - Paraspeckles } \\
\text { - Nucleoli } \\
\text { - PML bodies }\end{array}$ & $\begin{array}{l}\text { Amyloid bodies } \\
\text { Balbiani bodies }\end{array}$ \\
\hline Protein mobility & $\begin{array}{c}\text { Proteins are mobile; continuously exchanging } \\
\text { with the structure and the surrounding } \\
\text { milieu }\end{array}$ & $\begin{array}{l}\text { Proteins are immobile; engaged in } \\
\text { strong intermolecular interactions }\end{array}$ \\
\hline Shape & Spherical & Spherical \\
\hline Characteristics & $\begin{array}{l}\text { Structure is dynamic; exhibiting properties of } \\
\text { water droplets: } \\
\bullet \quad \text { Fluid } \\
\bullet \quad \text { Cycles of fusion (coalescence) and } \\
\text { fission } \\
\bullet \quad \text { Wetting behavior } \\
\text { Flows under shear force }\end{array}$ & $\begin{array}{c}\text { Structure is non-dynamic; } \\
\text { exhibiting properties of amyloids: } \\
\bullet \quad \text { Static } \\
\text { - Positive staining with } \\
\text { amyloidophilic dyes (e.g. Congo red) } \\
\text { - } \text { Resistant to Proteinase K } \\
\text { - Insoluble in common } \\
\text { - } \text { detergents } \\
\text { - Cross- } \beta \text { diffraction pattern } \\
\end{array}$ \\
\hline $\begin{array}{c}\text { Material } \\
\text { properties }\end{array}$ & Viscous & Elastic \\
\hline Function & Biochemical reactions & Cell dormancy \\
\hline Mechanism & Liquid-liquid phase separation & Liquid-to-solid phase transition \\
\hline
\end{tabular}

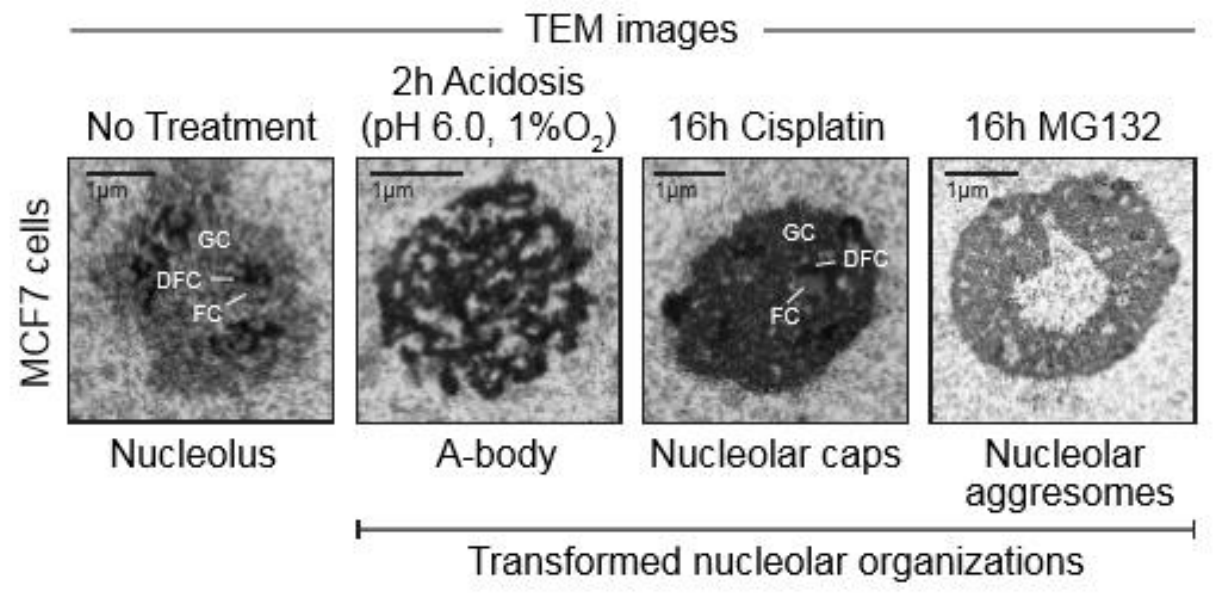

Figure 1. Contrasting A-bodies to other transformed nucleolar organizations, namely nucleolar caps and nucleolar aggresomes. Nucleoli can be transformed into A-bodies, nucleolar caps or nucleolar aggresomes under acidotic conditions, transcriptional arrest (16h cisplatin) and proteosomal inhibition (16h MG132), respectively. By transmission electron microscopy (TEM), A-bodies appear 
fibrillar and distinct from the amorphous, electron-dense nucleolar structure observed when nucleolar caps form or from the electron-light nucleolar cavity that is a nucleolar aggresome. Far right image taken from [67].

\section{Protein sequestration into A-bodies: an example of physiological liquid-to-solid phase transition}

At any one time, under the microscope, a plethora of membrane-less compartments populate the cell. How compartments maintain their unique identities and how proteins and/or RNA discriminate in which compartment they will be found has yet to be clarified. New insights into the biophysical properties of membrane-less compartments have demonstrated unexpected links between the sequence-encoded information in protein and RNA components of compartments and the material properties they impart, in dictating function [59,78-86]. A flurry of recent studies demonstrated the importance of RNA-binding proteins with prion-like, low complexity domains (LCDs) in forming membrane-less compartments. Equally as exciting has been the work done to uncover the specific sequences or structural elements embedded in architectural RNA that dictate the biophysical properties of membrane-less compartments [87,88]. With these new tools, we have begun to understand how specific elements within essential RNA and protein components are involved in A-body biogenesis.

\section{A multi-step model of A-body biogenesis}

Time-lapse imaging of A-body formation revealed that the solid-state organization is achieved through what appears to be a liquid-to-solid phase transition, in which proteins are initially found in liquid-like droplets that mature into a solid-like structure. These liquid-like droplets are distinct from the established nucleolar compartments involved in ribosome biosynthesis [89]. It was Woodruff et al. [90] who first proposed that solid-like structures such as A-bodies and Balbiani bodies originate from a liquid-liquid phase separation event that matures over time into a solid-like state. Fig. 2 is a step-wise reference guide of A-body formation and disassembly, showcasing A-body biogenesis as a precisely choreographed routine rather than simply a means to store misfolded aggregates.



Figure 2. Amyloid body biogenesis is a precisely choreographed routine. We propose that, on stress, low complexity rIGSRNA derived from the rDNA intergenic spacer accumulate in the nucleolus. Step one: Low complexity rIGSRNA interact with short cationic peptides, such as the R/H-rich sequence of the ACM (formally NoDS), to form nucleolar liquid-like foci. Step two: Local concentration of proteins with amyloidogenic propensity in the liquid droplets triggers physiological amyloidogenesis and generates nascent A-bodies. Step three: Once seeded, nascent A-bodies self-assemble into fibrillar, solid-like A-bodies. A-bodies enable cells to rapidly and reversibly store a large array of proteins and enter cellular dormancy in response to stress. Step four: Upon recovery/stimulus termination, A-body disaggregation is mediated by heat-shock protein (hsp) chaperones 70 and 90 . Through these steps, A-body biogenesis represents a physiological liquid-to-solid phase transition.

Low complexity rIGSRNA form complex coacervates with the R/H-rich domain of the NoDS 
What drives the formation of the initial liquid-liquid phase separation event in the nucleolus? In mammals, the nucleolus is organized around a scaffold of $~ 400$ ribosomal DNA (rDNA) tandem repeats of $43 \mathrm{~kb}[8,91]$. Each repeat consists of an rDNA enhancer/promoter located directly upstream of its ribosomal RNA (rRNA) genes separated by an intergenic spacer (rIGS) of variable length and organization [92,93] (Fig. 3A). The rIGS is an enigmatic region of the human genome historically, and erroneously, called the "non-transcribed region" [92]. Interestingly, in recent years, species conservation [94] and functional studies have demonstrated that these regions of the genome are transcriptionally active, known to generate noncoding RNA (ncRNA) involved in regulating rRNA expression [95-100], controlling PTBP1-regulated alternative splicing [101], and assembling A-bodies [26,27] (Fig. 3A).

The appearance of liquid droplets in the nucleolus coincides with the induction of long noncoding RNA originating from the rIGS, appropriately referred to as rIGSRNA (ribosomal intergenic spacer RNA) [27]. For example, in response to acidosis, the rDNA intergenic spacer at position $28 \mathrm{~kb}$ produces IGS $_{28} \mathrm{RNA}$ that is required for immobilization of proteins in A-bodies. Likewise, heat shock triggers the production of rIGS ${ }_{16} \mathrm{RNA}_{\mathrm{N}}$ and $\mathrm{rGS}_{22} \mathrm{RNA}$. Recent work suggests that low complexity dinucleotide repetitive sequences operate as the functional determinants of rIGSRNA to recruit proteins to A-bodies [89] (Fig. 3B). Live cell imaging and in vitro assays indicate that negatively-charged low complexity sequences of rIGSRNA co-assemble with positively-charged $\mathrm{R} / \mathrm{H}$-rich peptide domains of the NoDS more efficiently than with high complexity sequences, to form dense liquid droplets in the nucleolus [89]. The interaction between low complexity RNA and the $\mathrm{R} / \mathrm{H}$-rich region of the NoDS is likely driven by complex coacervation, a form of liquid-liquid phase separation based on electrostatic attraction that has been observed in other cellular settings in vivo [83,85]. It has been suggested that unstructured low complexity RNA molecules are more efficient than their structured high complexity counterparts at driving complex coacervation because the random coil state provides greater accessibility for multivalent interactions with short cationic molecules [102].

A

Pre-rRNA (13.7kb)

B
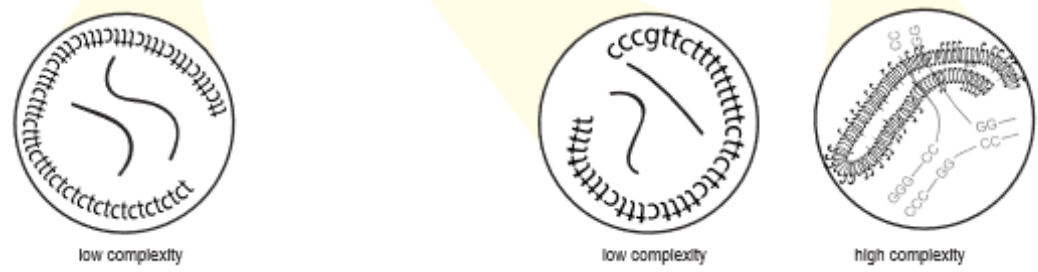

Figure 3. Induction of noncoding RNA (ncRNA) from the ribosomal cassette. A: Schematic of a single human rDNA repeat unit, which is composed of a $\sim 13 \mathrm{~kb}$ pre-rRNA coding region flanked by a $\sim 30 \mathrm{~kb}$ intergenic spacer (rIGS). The rIGS transcribes several functional noncoding RNA. Stimuli-specific loci of rIGS produce rIGSRNA (ribosomal InterGenic Spacer RNA) required for A-body formation. rIGS28RNA and rIGS16RNA/rIGS22RNA are produced under acidotic (yellow) and heat shock (red) conditions, respectively. No function has been ascribed to rIGS18RNA yet. Other ncRNA found in the rIGS include a $>10 \mathrm{~kb}$ transcript called PNCTR (pyrimidine-rich noncoding transcript) involved in PTBP1-binding (purple), pRNA (green) and anti-sense PAPAS (brown) involved in rRNA regulation, as well as Alu element-derived (grey boxes) RNA involved in nucleolar architecture. B: rIGSRNA contain low complexity sequences comprising of long dinucleotide repeats, as determined by RNA 
sequencing, RT cloning and RNA-FISH. This is in contrast to other ncRNA that display high complexity, i.e. possess secondary structure, such as pRNA.

Amyloidogenic properties of the NoDS trigger the liquid-to-solid transition

In principle, solid-like structures should be composed of proteins that possess fibrillation propensity, i.e. increased likelihood of forming fibrils. Bioinformatic analysis of the consensus NoDS revealed the hydrophobic LXV motifs make up a region of high fibrillation propensity, as predicted by ZipperDB based on a Rosetta energy score of less than $-23 \mathrm{kcal} / \mathrm{mol}$. As a whole, the NoDS bears a striking resemblance to that of the prototypical pathological $\beta$-amyloid associated with Alzheimer's disease, in which a R/H-rich sequence is in close proximity to a highly amyloidogenic stretch referred to in literature as the P3 fragment. The fibrillation propensity of the NoDS was validated by demonstrating it exhibited classic amyloidogenic properties previously observed for $\beta$-amyloid, namely a cross- $\beta$ x-ray diffraction pattern and formation of fibrils in vitro. For these reasons, the NoDS has been renamed the Amyloid-Converting Motif (ACM) (Fig. 4A).

It has been proposed that the function of the initial liquid state is to locally concentrate proteins with fibrillation propensity that would otherwise be at levels below the critical threshold in the cell [58]. Concentration-dependent activation of protein fibrillation has been well-documented in vitro [58,103]. Indeed, markers of early or nascent A-bodies included nuclear ANS and A11 staining [89], typically used in vitro to indicate accumulating pre-amyloidogenic structures [104-106]. Once seeded, the amyloidogenic process can be spontaneously triggered. Nascent A-bodies have been observed to expand by self-assembly, in which soluble proteins are added directly and autonomously to the growing amyloid structure [89].

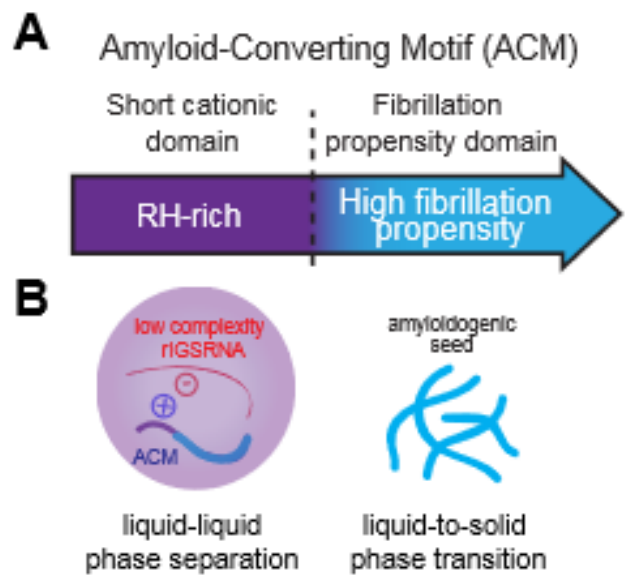

Figure 4. The Amyloid-Converting Motif (ACM). A: The ACM is necessary and sufficient to target and immobilize proteins in A-bodies. It consists of a R/H-rich short cationic domain flanking a high fibrillation propensity domain (determined by Rosetta energy of < $-23 \mathrm{kcal} / \mathrm{mol}$ ). B: Its bipartite nature allows the ACM to traverse phase boundaries. Complex coacervation of short cationic domains and low complexity rIGSRNA (liquid-liquid phase separation) concentrates fibrillation propensity domains to activate a liquid-to-solid phase transition to form A-bodies.

The ACM traverses across phase boundaries to confer A-body identity

As described above, low complexity RNA sequences are important in conferring A-bodies their unique identity [89], adding to the list of architectural determinants, which includes sequence-specific RNA [107], mRNA secondary structure [70,79] and short unstructured RNA in vitro [108]. However, not every protein containing positively charged domains will be found in A-bodies. The low complexity rIGSRNA-mediated coacervates themselves appear immiscible from the three canonical compartments of the nucleolus [44,89], suggesting they exhibit distinct biophysical properties that may exclude mixing. Ultimately, it is the bipartite nature of the ACM that confers A-body identity 
and differentiates the ACM from other motifs. The R/H-rich "short cationic domain" mediates liquidliquid phase separation while the "fibrillation propensity domain" initiates the liquid-to-solid phase transition [26,89] (Fig. 4B). In this model, proteins without a region of high fibrillation propensity are unlikely to be incorporated into A-bodies. The balance of positive to negative charge ratio, presence of polar and/or aromatic residues, and modifications $[109,110]$ will affect how proteins engage in intermolecular interactions with other proteins and RNA. Therefore, the ACM is versatile as it can physiologically transition proteins across phase boundaries.

\section{Physiological amyloids promote cell dormancy}

Endogenous A-bodies have been found in primary cultures and cell lines exposed to various stimuli, the cores of human tumors, and subsets of cells in normal human and mouse tissues [26]. So, while A-bodies share structural features with pathological amyloids, their ubiquitous and reversible nature is indicative of a physiological function. Pathway enrichment analysis determined that many of the proteins sequestered in A-bodies are involved in cell cycle progression and DNA synthesis, such as CDK1, POLD1, DNMT1 [26]. By temporarily detaining key factors from their sites of activity into A-bodies, major molecular networks are disrupted, resulting in metabolic inactivity [77] and arrested proliferation/DNA synthesis [26]. This is different from a quiescent state (G0) in the cell cycle where cells arrest proliferation but remain metabolically active. Therefore, the biological role of Abody formation is to promote cellular dormancy as an adaptive response to environmental stressors. These findings are consistent with reports that show Balbiani bodies [73] and fluid-to-solid transitions in yeast [74] promote dormancy, reinforcing the concept that cells utilize different states of matter to perform various biological functions (Table 2). The material properties of solid-like systems - nondynamic, amyloid-like - result in loss of metabolic activity and promote cellular dormancy while those of liquid-like systems - dynamic, fluid-like - concentrate biochemical reactions.

\section{A-bodies as a target for therapeutic discovery}

Implications for pathological amyloids

A-bodies that are physiologically produced remain confined within the nucleus. How the nuclear environment alters the interaction properties of aggregation-prone proteins to prevent toxicity $[111,112]$ compared to the cytoplasm is unclear. These results imply the cell tightly regulates the induction and degradation of low complexity rIGSRNA within the nucleolus in response to stimuli. Whether aberrant production or localization of low complexity RNA are involved in amyloid pathologies such as Alzheimer's and Huntington's Diseases will be the subject of future studies.

\section{Implications for tumor cell dormancy and metastasis}

Given the evidence that A-bodies promote cell dormancy, perhaps there is a link between Abody formation and tumor cell dormancy, that allow cancer cells to adapt to the harsh hypoxic/acidotic conditions of the tumor microenvironment. Such a connection has pertinent implications for chemotherapeutic resistance and metastasis [113,114]. With major metabolic pathways shut down and key drug targets stored away in A-bodies, a small population of dormant tumor cells may be resistent to treatment, survive for a prolonged period of time and contribute to disease recurrence or metastasis upon their metabolic reactivation. Preventing cancer cells from forming A-bodies and going dormant or, more realistically, preventing cancer cells from reactivating may become a viable treatment option.

An interesting avenue of research that may offer a more effective cancer treatment strategy involves manipulating the proteins that evade capture into A-bodies. Interestingly, proteins that remain active to sustain basal metabolism and viability under stress tend to be devoid of fibrillation propensity domains and evade capture by A-bodies. These proteins would be more susceptible to manipulation and serve as better chemotherapeutic targets than proteins captured in A-bodies.

\section{Conclusion}


Twenty-years after its discovery, the study of nucleolar sequestration has led to the discovery of a physiological amyloidogenic program that converts the liquid nucleolus into the solid A-body. The study of A-bodies has provided a rare opportunity to interrogate the functional and regulatory interactions necessary to form amyloids in a cellular setting. Why exactly nucleolar coacervates differ from other liquid condensates in their ability to transition to the solid state remains a fundamental question that will surely offer alternative insights into how we understand and treat diseases related to age, neurodegeneration and cancer.

Funding: This work was supported by grants from the National Institute of General Medical Sciences (R01GM115342) (S.L.), the National Cancer Institute (R01CA200676) of the NIH, and the Sylvester Comprehensive Cancer Center (S.L.).

Conflicts of interest: The authors declare no conflict of interest.

\section{References}

1. Miller, O.L., Jr. and B.R. Beatty, Visualization of nucleolar genes. Science, 1969. 164(3882): p. 955-7.

2. Perry, R.P., On the nucleolar and nuclear dependence of cytoplasmic RNA synthesis in HeLa cells. Exp Cell Res, 1960. 20: p. 216-20.

3. Perry, R.P., The Cellular Sites of Synthesis of Ribosomal and 4s Rna. Proc Natl Acad Sci U S A, 1962. 48(12): p. 2179-86.

4. Hernandez-Verdun, D., Nucleolus: from structure to dynamics. Histochem Cell Biol, 2006. 125(1-2): p. 127-37.

5. Raska, I., P.J. Shaw, and D. Cmarko, Structure and function of the nucleolus in the spotlight. Curr Opin Cell Biol, 2006. 18(3): p. 325-34.

6. Pederson, T., The nucleolus. Cold Spring Harb Perspect Biol, 2011. 3(3).

7. Boisvert, F.M., et al., The multifunctional nucleolus. Nat Rev Mol Cell Biol, 2007. 8(7): p. 574-85.

8. Nemeth, A. and I. Grummt, Dynamic regulation of nucleolar architecture. Curr Opin Cell Biol, 2018. 52: p. 105-111.

9. Pederson, T. and R.Y. Tsai, In search of nonribosomal nucleolar protein function and regulation. J Cell Biol, 2009. 184(6): p. 771-6.

10. Boulon, S., et al., The nucleolus under stress. Mol Cell, 2010. 40(2): p. 216-27.

11. Emmott, E. and J.A. Hiscox, Nucleolar targeting: the hub of the matter. EMBO Rep, 2009. 10(3): p. 231-8.

12. Bachant, J.B. and S.J. Elledge, Mitotic treasures in the nucleolus. Nature, 1999. 398(6730): p. 757-8.

13. Bernardi, R., et al., PML regulates p53 stability by sequestering Mdm2 to the nucleolus. Nat Cell Biol, 2004. 6(7): p. 665-72.

14. Lohrum, M.A., et al., Identification of a cryptic nucleolar-localization signal in MDM2. Nat Cell Biol, 2000. 2(3): p. 179-81.

15. Shou, W., et al., Exit from mitosis is triggered by Tem1-dependent release of the protein phosphatase Cdc14 from nucleolar RENT complex. Cell, 1999. 97(2): p. 233-44.

16. Visintin, R., E.S. Hwang, and A. Amon, Cfi1 prevents premature exit from mitosis by anchoring Cdc14 phosphatase in the nucleolus. Nature, 1999. 398(6730): p. 818-23.

17. Weber, J.D., et al., Nucleolar Arf sequesters Mdm2 and activates p53. Nat Cell Biol, 1999. 1(1): p. 206.

18. Mekhail, K., et al., HIF activation by pH-dependent nucleolar sequestration of VHL. Nat Cell Biol, 2004. 6(7): p. 642-7.

19. San-Segundo, P.A. and G.S. Roeder, Pch2 links chromatin silencing to meiotic checkpoint control. Cell, 1999. 97(3): p. 313-24.

20. Chen, X., et al., Tissue-specific TAFs counteract Polycomb to turn on terminal differentiation. Science, 2005. 310(5749): p. 869-72.

21. Martindill, D.M., et al., Nucleolar release of Hand1 acts as a molecular switch to determine cell fate. Nat Cell Biol, 2007. 9(10): p. 1131-41.

22. Chen, J. and L.A. Stark, Aspirin Prevention of Colorectal Cancer: Focus on NF-kappaB Signalling and the Nucleolus. Biomedicines, 2017. 5(3). 
23. Stark, L.A. and M.G. Dunlop, Nucleolar sequestration of RelA (p65) regulates NF-kappaB-driven transcription and apoptosis. Mol Cell Biol, 2005. 25(14): p. 5985-6004.

24. Kurki, S., L. Latonen, and M. Laiho, Cellular stress and DNA damage invoke temporally distinct Mdm2, p53 and PML complexes and damage-specific nuclear relocalization. J Cell Sci, 2003. 116(Pt 19): p. 3917-25.

25. Wong, J.M., L. Kusdra, and K. Collins, Subnuclear shuttling of human telomerase induced by transformation and DNA damage. Nat Cell Biol, 2002. 4(9): p. 731-6.

26. Audas, T.E., et al., Adaptation to Stressors by Systemic Protein Amyloidogenesis. Dev Cell, 2016. 39(2): p. $155-168$.

27. Audas, T.E., M.D. Jacob, and S. Lee, Immobilization of proteins in the nucleolus by ribosomal intergenic spacer noncoding RNA. Mol Cell, 2012. 45(2): p. 147-57.

28. Jacob, M.D., et al., Environmental cues induce a long noncoding RNA-dependent remodeling of the nucleolus. Mol Biol Cell, 2013. 24(18): p. 2943-53.

29. Messaoudi, L., et al., Subcellular distribution of human RDM1 protein isoforms and their nucleolar accumulation in response to heat shock and proteotoxic stress. Nucleic Acids Res, 2007. 35(19): p. 657187.

30. Banski, P., et al., Nucleolar targeting of the chaperone hsc70 is regulated by stress, cell signaling, and a composite targeting signal which is controlled by autoinhibition. J Biol Chem, 2010. 285(28): p. 2185867.

31. Moore, H.M., et al., Quantitative proteomics and dynamic imaging of the nucleolus reveal distinct responses to UV and ionizing radiation. Mol Cell Proteomics, 2011. 10(10): p. M111 009241.

32. Scott, M., et al., UV induces nucleolar translocation of ING1 through two distinct nucleolar targeting sequences. Nucleic Acids Res, 2001. 29(10): p. 2052-8.

33. Sydorskyy, Y., et al., A novel mechanism for SUMO system control: regulated Ulp1 nucleolar sequestration. Mol Cell Biol, 2010. 30(18): p. 4452-62.

34. Wang, L., et al., The nucleolus and viral infection. Virol Sin, 2010. 25(3): p. 151-7.

35. Andersen, J.S., et al., Nucleolar proteome dynamics. Nature, 2005. 433(7021): p. 77-83.

36. Sansam, C.L., K.S. Wells, and R.B. Emeson, Modulation of RNA editing by functional nucleolar sequestration of ADAR2. Proc Natl Acad Sci U S A, 2003. 100(24): p. 14018-23.

37. Latonen, L., Nucleolar aggresomes as counterparts of cytoplasmic aggresomes in proteotoxic stress. Proteasome inhibitors induce nuclear ribonucleoprotein inclusions that accumulate several key factors of neurodegenerative diseases and cancer. Bioessays, 2011. 33(5): p. 386-95.

38. Latonen, L., et al., Proteasome inhibitors induce nucleolar aggregation of proteasome target proteins and polyadenylated RNA by altering ubiquitin availability. Oncogene, 2011. 30(7): p. 790-805.

39. Arabi, A., Accumulation of c-Myc and proteasomes at the nucleoli of cells containing elevated c-Myc protein levels. Journal of Cell Science, 2003. 116(9): p. 1707-1717.

40. Misteli, T., Physiological importance of RNA and protein mobility in the cell nucleus. Histochem Cell Biol, 2008. 129(1): p. 5-11.

41. Phair, R.D. and T. Misteli, High mobility of proteins in the mammalian cell nucleus. Nature, 2000. 404(6778): p. 604-9.

42. Lippincott-Schwartz, J., N. Altan-Bonnet, and G.H. Patterson, Photobleaching and photoactivation: following protein dynamics in living cells. Nat Cell Biol, 2003. Suppl: p. S7-14.

43. Dundr, M., et al., In vivo kinetics of Cajal body components. J Cell Biol, 2004. 164(6): p. 831-42.

44. Feric, M., et al., Coexisting Liquid Phases Underlie Nucleolar Subcompartments. Cell, 2016. 165(7): p. 1686-97.

45. Brangwynne, C.P., T.J. Mitchison, and A.A. Hyman, Active liquid-like behavior of nucleoli determines their size and shape in Xenopus laevis oocytes. Proc Natl Acad Sci U S A, 2011. 108(11): p. 4334-9.

46. Mekhail, K., et al., Regulation of ubiquitin ligase dynamics by the nucleolus. J Cell Biol, 2005. 170(5): p. 733-44.

47. Tomson, B.N., et al., Regulation of Spo12 phosphorylation and its essential role in the FEAR network. Curr Biol, 2009. 19(6): p. 449-60. 
48. Mekhail, K., et al., Identification of a common subnuclear localization signal. Mol Biol Cell, 2007. 18(10): p. 3966-77.

49. Mikhaleva, E.A., et al., The nucleolar transcriptome regulates Piwi shuttling between the nucleolus and the nucleoplasm. Chromosome Res, 2018.

50. Audas, T.E., M.D. Jacob, and S. Lee, The nucleolar detention pathway: A cellular strategy for regulating molecular networks. Cell Cycle, 2012. 11(11): p. 2059-62.

51. Weber, J.D., et al., Cooperative signals governing ARF-mdm2 interaction and nucleolar localization of the complex. Mol Cell Biol, 2000. 20(7): p. 2517-28.

52. Tao, W. and A.J. Levine, P19(ARF) stabilizes p53 by blocking nucleo-cytoplasmic shuttling of Mdm2. Proc Natl Acad Sci U S A, 1999. 96(12): p. 6937-41.

53. Poyurovsky, M.V., et al., Nucleotide binding by the Mdm2 RING domain facilitates Arf-independent Mdm2 nucleolar localization. Mol Cell, 2003. 12(4): p. 875-87.

54. Mattsson, K., et al., Proteins associated with the promyelocytic leukemia gene product (PML)-containing nuclear body move to the nucleolus upon inhibition of proteasome-dependent protein degradation. Proc Natl Acad Sci U S A, 2001. 98(3): p. 1012-7.

55. Azzam, R., et al., Phosphorylation by cyclin B-Cdk underlies release of mitotic exit activator Cdc14 from the nucleolus. Science, 2004. 305(5683): p. 516-9.

56. Berchowitz, L.E., et al., Regulated Formation of an Amyloid-like Translational Repressor Governs Gametogenesis. Cell, 2015. 163(2): p. 406-18.

57. Kayatekin, C., et al., Prion-like proteins sequester and suppress the toxicity of huntingtin exon 1. Proc Natl Acad Sci U S A, 2014. 111(33): p. 12085-90.

58. Knowles, T.P., M. Vendruscolo, and C.M. Dobson, The amyloid state and its association with protein misfolding diseases. Nat Rev Mol Cell Biol, 2014. 15(6): p. 384-96.

59. Shin, Y. and C.P. Brangwynne, Liquid phase condensation in cell physiology and disease. Science, 2017. 357(6357).

60. Banani, S.F., et al., Biomolecular condensates: organizers of cellular biochemistry. Nat Rev Mol Cell Biol, 2017. 18(5): p. 285-298.

61. Weber, S.C. and C.P. Brangwynne, Getting RNA and protein in phase. Cell, 2012. 149(6): p. $1188-91$.

62. Protter, D.S. and R. Parker, Principles and Properties of Stress Granules. Trends Cell Biol, 2016. 26(9): p. 668-79.

63. Zhu, L. and C.P. Brangwynne, Nuclear bodies: the emerging biophysics of nucleoplasmic phases. Curr Opin Cell Biol, 2015. 34: p. 23-30.

64. Brangwynne, C.P., et al., Germline $P$ granules are liquid droplets that localize by controlled dissolution/condensation. Science, 2009. 324(5935): p. 1729-32.

65. Lyons, S.M. and P. Anderson, RNA-Seeded Functional Amyloids Balance Growth and Survival. Dev Cell, 2016. 39(2): p. 131-132.

66. Woodruff, J.B., A.A. Hyman, and E. Boke, Organization and Function of Non-dynamic Biomolecular Condensates. Trends Biochem Sci, 2018. 43(2): p. 81-94.

67. Patel, A., et al., A Liquid-to-Solid Phase Transition of the ALS Protein FUS Accelerated by Disease Mutation. Cell, 2015. 162(5): p. 1066-77.

68. Peskett, T.R., et al., A Liquid to Solid Phase Transition Underlying Pathological Huntingtin Exon1 Aggregation. Mol Cell, 2018. 70(4): p. 588-601 e6.

69. Posey, A.E., et al., Profilin reduces aggregation and phase separation of huntingtin N-terminal fragments by preferentially binding to soluble monomers and oligomers. J Biol Chem, 2018. 293(10): p. 3734-3746.

70. Jain, A. and R.D. Vale, RNA phase transitions in repeat expansion disorders. Nature, 2017. 546(7657): p. 243-247.

71. Mateju, D., et al., An aberrant phase transition of stress granules triggered by misfolded protein and prevented by chaperone function. EMBO J, 2017. 36(12): p. 1669-1687.

72. Murakami, T., et al., ALS/FTD Mutation-Induced Phase Transition of FUS Liquid Droplets and Reversible Hydrogels into Irreversible Hydrogels Impairs RNP Granule Function. Neuron, 2015. 88(4): p. 678-90.

73. Boke, E., et al., Amyloid-like Self-Assembly of a Cellular Compartment. Cell, 2016. 166(3): p. 637-50. 
74. Munder, M.C., et al., A pH-driven transition of the cytoplasm from a fluid- to a solid-like state promotes entry into dormancy. Elife, 2016. 5.

75. Shav-Tal, Y., et al., Dynamic sorting of nuclear components into distinct nucleolar caps during transcriptional inhibition. Mol Biol Cell, 2005. 16(5): p. 2395-413.

76. Kruger, T. and U. Scheer, p53 localizes to intranucleolar regions distinct from the ribosome production compartments. J Cell Sci, 2010. 123(Pt 8): p. 1203-8.

77. Mekhail, K., et al., Restriction of $r$ RNA synthesis by VHL maintains energy equilibrium under hypoxia. Cell Cycle, 2006. 5(20): p. 2401-13.

78. Weber, S.C., Sequence-encoded material properties dictate the structure and function of nuclear bodies. Curr Opin Cell Biol, 2017. 46: p. 62-71.

79. Langdon, E.M., et al., mRNA structure determines specificity of a polyQ-driven phase separation. Science, 2018. 360(6391): p. 922-927.

80. Kato, M., et al., Cell-free formation of RNA granules: low complexity sequence domains form dynamic fibers within hydrogels. Cell, 2012. 149(4): p. 753-67.

81. Molliex, A., et al., Phase separation by low complexity domains promotes stress granule assembly and drives pathological fibrillization. Cell, 2015. 163(1): p. 123-33.

82. Brangwynne, Clifford P., P. Tompa, and Rohit V. Pappu, Polymer physics of intracellular phase transitions. Nature Physics, 2015. 11(11): p. 899-904.

83. Pak, C.W., et al., Sequence Determinants of Intracellular Phase Separation by Complex Coacervation of a Disordered Protein. Mol Cell, 2016. 63(1): p. 72-85.

84. Nott, T.J., et al., Phase transition of a disordered nuage protein generates environmentally responsive membraneless organelles. Mol Cell, 2015. 57(5): p. 936-47.

85. Altmeyer, M., et al., Liquid demixing of intrinsically disordered proteins is seeded by poly(ADP-ribose). Nat Commun, 2015. 6: p. 8088.

86. Elbaum-Garfinkle, S., et al., The disordered P granule protein LAF-1 drives phase separation into droplets with tunable viscosity and dynamics. Proc Natl Acad Sci U S A, 2015. 112(23): p. 7189-94.

87. Chujo, T. and T. Hirose, Nuclear Bodies Built on Architectural Long Noncoding RNAs: Unifying Principles of Their Construction and Function. Mol Cells, 2017. 40(12): p. 889-896.

88. Chujo, T., et al., Unusual semi-extractability as a hallmark of nuclear body-associated architectural noncoding RNAs. EMBO J, 2017. 36(10): p. 1447-1462.

89. Wang, M., et al., Stress-Induced Low Complexity RNA Activates Physiological Amyloidogenesis. Cell Rep, 2018. 24(7): p. 1713-1721 e4.

90. Woodruff, J.B., et al., The Centrosome Is a Selective Condensate that Nucleates Microtubules by Concentrating Tubulin. Cell, 2017. 169(6): p. 1066-1077 e10.

91. Sharifi, S. and H. Bierhoff, Regulation of RNA Polymerase I Transcription in Development, Disease, and Aging. Annu Rev Biochem, 2018.

92. Smirnov, E., et al., Nucleolar DNA: the host and the guests. Histochem Cell Biol, 2016. 145(4): p. 35972.

93. Gonzalez, I.L. and J.E. Sylvester, Complete sequence of the 43-kb human ribosomal DNA repeat: analysis of the intergenic spacer. Genomics, 1995. 27(2): p. 320-8.

94. Agrawal, S. and A.R.D. Ganley, The conservation landscape of the human ribosomal RNA gene repeats. PLoS One, 2018. 13(12): p. e0207531.

95. Mayer, C., M. Neubert, and I. Grummt, The structure of NoRC-associated RNA is crucial for targeting the chromatin remodelling complex NoRC to the nucleolus. EMBO Rep, 2008. 9(8): p. 774-80.

96. Schmitz, K.M., et al., Interaction of noncoding RNA with the $r D N A$ promoter mediates recruitment of DNMT3b and silencing of rRNA genes. Genes Dev, 2010. 24(20): p. 2264-9.

97. Mayer, C., et al., Intergenic transcripts regulate the epigenetic state of rRNA genes. Mol Cell, 2006. 22(3): p. 351-61.

98. Zhao, Z., et al., IncRNA-Induced Nucleosome Repositioning Reinforces Transcriptional Repression of rRNA Genes upon Hypotonic Stress. Cell Rep, 2016. 14(8): p. 1876-82.

99. Zhao, Z., et al., Heat shock represses $r R N A$ synthesis by inactivation of TIF-IA and lncRNA-dependent changes in nucleosome positioning. Nucleic Acids Res, 2016. 44(17): p. 8144-52. 
100.Zhao, Z., et al., IncRNA PAPAS tethered to the rDNA enhancer recruits hypophosphorylated CHD4/NuRD to repress rRNA synthesis at elevated temperatures. Genes Dev, 2018. 32(11-12): p. 836848.

101.Yap, K., et al., A Short Tandem Repeat-Enriched RNA Assembles a Nuclear Compartment to Control Alternative Splicing and Promote Cell Survival. Mol Cell, 2018. 72(3): p. 525-540 e13.

102. Aumiller, W.M., Jr., et al., RNA-Based Coacervates as a Model for Membraneless Organelles: Formation, Properties, and Interfacial Liposome Assembly. Langmuir, 2016. 32(39): p. 10042-10053.

103.Eisenberg, D. and M. Jucker, The amyloid state of proteins in human diseases. Cell, 2012. 148(6): p. 1188-203.

104.Hawe, A., M. Sutter, and W. Jiskoot, Extrinsic fluorescent dyes as tools for protein characterization. Pharm Res, 2008. 25(7): p. 1487-99.

105.Tang, Z., et al., MEK guards proteome stability and inhibits tumor-suppressive amyloidogenesis via HSF1. Cell, 2015. 160(4): p. 729-44.

106.Kayed, R., et al., Fibril specific, conformation dependent antibodies recognize a generic epitope common to amyloid fibrils and fibrillar oligomers that is absent in prefibrillar oligomers. Mol Neurodegener, 2007. 2: p. 18.

107.Yamazaki, T., et al., Functional Domains of NEAT1 Architectural lncRNA Induce Paraspeckle Assembly through Phase Separation. Mol Cell, 2018. 70(6): p. 1038-1053 e7.

108.Nott, T.J., T.D. Craggs, and A.J. Baldwin, Membraneless organelles can melt nucleic acid duplexes and act as biomolecular filters. Nat Chem, 2016. 8(6): p. 569-75.

109.Aumiller, W.M., Jr. and C.D. Keating, Phosphorylation-mediated RNA/peptide complex coacervation as a model for intracellular liquid organelles. Nat Chem, 2016. 8(2): p. 129-37.

110.Monahan, Z., et al., Phosphorylation of the FUS low-complexity domain disrupts phase separation, aggregation, and toxicity. EMBO J, 2017. 36(20): p. 2951-2967.

111. Maharana, S., et al., RNA buffers the phase separation behavior of prion-like RNA binding proteins. Science, 2018. 360(6391): p. 918-921.

112. Woerner, A.C., et al., Cytoplasmic protein aggregates interfere with nucleocytoplasmic transport of protein and RNA. Science, 2016. 351(6269): p. 173-6.

113.Li, J., et al., Dormant Cells: The Original Cause of Tumor Recurrence and Metastasis. Cell Biochem Biophys, 2015. 72(2): p. 317-20.

114.Li, S., et al., Model of tumor dormancy/recurrence after short-term chemotherapy. PLoS One, 2014. 9(5): p. e98021. 